\title{
Correspondence
}

\section{Known but unpredictable - an argument for complexity}

Since the seminal paper of Pokorny in $1983,{ }^{1}$ the prediction of suicides has not improved, as Large et al have pointed out in their current paper ${ }^{2}$ and in previous meta-analyses. ${ }^{3-7}$ In opposition to most current recommendations in suicide prevention, which still require clinicians to formulate levels of suicide risk, ${ }^{8}$ Large et $a^{2}$ suggest that clinicians should give up risk formulation and instead focus directly on the individual needs of patients to deliver optimal care. They argue that uncertainty in the prediction of suicide is largely aleatory (dependent on random processes) and also epistemic (lacking knowledge). We think that one important explanation is missing: complexity.

Complexity refers to behaviours produced by nonlinear dynamic systems, which cannot be predicted in the long term, even if the generating system operates completely deterministically and is known in detail. The most prominent type of complex dynamics is deterministic chaos, which became familiar as the 'butterfly effect'. During chaotic dynamics, even the smallest differences in initial conditions lead to a massive divergence of trajectories over time. Owing to complex behaviours such as chaos, from a nonlinear dynamical perspective, the failure of long-term predictions of suicidal behaviour could be a consequence not only of incomplete epistemic knowledge (e.g. unspecific or unknown risk factors) or aleatory processes (random noise), but also of the inherent complexity of the underlying system.

Are there any alternatives for predicting suicidal behaviour from a nonlinear dynamical perspective? Natural sciences (e.g. geophysics) have developed methods for the short-term prediction of extreme events (e.g. tsunamis), based on continuous monitoring of appropriate signals and identification of nonlinear dynamical precursors. ${ }^{9,10}$ This might be a promising approach for suicide research as well. Given the recent improvements of scientific methods, an empirical application of complexity theory in suicide research seems realistic. ${ }^{11,12}$ However, it still has to be demonstrated that such novel approaches are feasible in clinical practice and that they can in fact improve the prediction of suicides.

We believe that suicidology needs to take complexity theory into consideration. If not, much time, effort and money will continue to go into approaches that, from the viewpoint of complexity theory, lead to a dead end. This includes the search for novel risk factors or combinations of risk factors (e.g. by applying machine learning) without acknowledging the underlying complex processes.

Martin Plöderl, Clinical Psychologist, and Clemens Fartacek, Clinical Psychologist, Department of Clinical Psychology and Department of Crisis Intervention and Suicide Prevention, Paracelsus Medical University, Salzburg, Austria; email: m.ploederl@salk.at

1 Pokorny AD. Prediction of suicide in psychiatric patients. Report of a prospective study. Arch Gen Psychiatry 1983; 40(3): 249-57.
2 Large M, Galletly C, Myles N, Ryan CJ, Myles H. Known unknowns and unknown unknowns in suicide risk assessment: evidence from meta-analyses of aleatory and epistemic uncertainty. BJPsych Bull 2017; 41(3): 160-3.

3 Chung DT, Ryan CJ, Hadzi-Pavlovic D, Singh SP, Stanton C, Large MM Suicide rates after discharge from psychiatric facilities: a systematic review and meta-analysis. JAMA Psychiatry 2017 74(7): 694-702.

4 Large M, Kaneson M, Myles N, Myles H, Gunaratne P, Ryan C. Meta-analysis of longitudinal cohort studies of suicide risk assessment among psychiatric patients: heterogeneity in results and lack of improvement over time. PLoS ONE 2016; 11(6): e0156322.

5 Large M, Myles N, Myles H, Corderoy A, Weiser M, Davidson M, et al. Suicide risk assessment among psychiatric inpatients: a systematic review and meta-analysis of high-risk categories. Psychol Med 2017; doi: 10.1017/S0033291717002537.

6 Large M, Sharma S, Cannon E, Ryan C, Nielssen O. Risk factors for suicide within a year of discharge from psychiatric hospital: a systematic meta-analysis. Aust N Z J Psychiatry 2011; 45(8): 619-28.

7 Walsh G, Sara G, Ryan CJ, Large M. Meta-analysis of suicide rates among psychiatric in-patients. Acta Psychiatr Scand 2015; 131(3): 17484.

8 Jacobs D, Brewer M. APA practice guideline. Provides recommendations for assessing and treating patients with suicidal behaviors. Psychiatr Ann 2004; 34(5): 373-80.

9 Albeverio S, Jentsch V, Kantz $\mathrm{H}$ (eds). Extreme Events in Nature and Society. Springer, 2006.

10 Albeverio S, Piterbarg V. Mathematical methods and concepts for the analysis of extreme events. In Extreme Events in Nature and Society (eds S Albeverio, V Jentsch, H Kantz). Springer, 2006.

11 Fartacek C, Schiepek G, Kunrath S, Fartacek R, Ploderl M. Real-time monitoring of non-linear suicidal dynamics: methodology and a demonstrative case report. Front Psychol 2016; 7: 130.

12 Schiepek G, Fartacek C, Sturm J, Kralovec K, Fartacek R, Ploderl M. Nonlinear dynamics: theoretical perspectives and application to suicidology. Suicide Life Threat Behav 2011; 41(6): 661-75.

doi:10.1192/bjb.2018.12

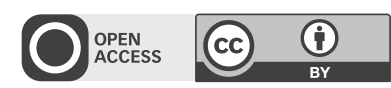

(c) The Authors 2018. This is an Open Access article, distributed under the terms of the Creative Commons Attribution licence (http://creativecommons.org/ licenses/by/4.0/), which permits unrestricted re-use, distribution, and reproduction in any medium, provided the original work is properly cited.

\section{Management of common mental disorders for psychogeriatric patients in Hong Kong: comparison of two clinics after 1 year of treatment}

We would like to update the findings of our pilot study which compared the enhanced common mental disorder clinic $(\mathrm{CMDC})^{1}$ and conventional specialist psychiatric out-patient clinic (SOPC) in the management of common mental disorders (CMDs) for psychogeriatric patients in our hospital in Hong Kong. In our previous letter to the editor, different clinical factors were compared between the two groups 6 months post-treatment. This time, findings for 1 year post-treatment were available. 
The CMDC is a 1-year programme with multidisciplinary involvement. There were 30 patients in each group. After 1 year of treatment, only 15 patients $(50 \%)$ remained in the CMDC, while 23 remained in the SOPC $(P=0.03)$. Ten patients (33.3\%) completed the CMDC programme and were successfully discharged from the CMDC. They did not require any medication for their CMDs. One patient refused to attend medical follow-up. One patient was transferred from CMDC to SOPC, as she was found to have dementia. Another patient was transferred to the general out-patient clinic for continuation of treatment for her mixed anxiety and depressive disorder.

Concerning psychological intervention, half of the patients in the CMDC group (50\%) were referred to a clinical psychologist, and ten had good adherence to appointments. For the SOPC group, only three patients $(10 \%)$ were referred to a clinical psychologist $(P<0.05)$.

Concerning antidepressant use, the rate at 1-year follow-up was 11 (36.7\%) for CMDC v. 20 (69\%) for SOPC patients $(P=0.02)$. Profiles of benzodiazepine and hypnotic use and prescription were similar prior to consultation, after the first consultation, 6 months post-treatment and 1 year post-treatment. Eight patients (26.7\%) in each group did not require benzodiazepines or hypnotics after the first consultation. The reduction in benzodiazepines and hypnotics was statistically significant $(P=0.04)$. At 1 year after treatment, ten patients $(33.3 \%)$ in each group were taking benzodiazepines or hypnotics.

In summary, psychiatrists of both clinics were able to reduce benzodiazepine and hypnotic use after the patients formally presented to the psychiatric clinic. Nearly half of the patients who were given a benzodiazepine or hypnotic did not require it afterwards. Instead, about two-thirds of them were treated with antidepressants. Significantly more patients did not require antidepressants at 1 year post-treatment in the CMDC group. There were also significantly more patients who did not require medical follow-up at 1 year for the CMDC group.

Remission of CMDs is possible for patients who have completed the 1-year CMDC. Its psychological and pharmacological components, as well as timely interventions, have contributed to its success. The treating team and the patients are aware of the time-limited nature of the programme and have expectations that suitable cases can be discharged from the programme upon its completion. This helps to ensure that the clinic is not overwhelmed by continual accumulation of cases. On the contrary, the SOPC does not have a specified duration of treatment and the doctors are less ready to discharge patients from the clinic if they are stable on medical treatment. It is not common to refer back stable cases to primary care. Enhanced collaboration between the SOPC and primary care in the management of CMDs can help to reduce the burden on the SOPC, so that it can have more capacity to deal with complicated and unstable cases. ${ }^{2}$ The acceptability of psychological interventions is expected to be better if they can be more tailored to elderly patients; in fact, many older people expressed a preference for talking therapies. ${ }^{3}$

Mimi Mei Cheung Wong, Psychiatrist, Pui-fai Pang, Psychiatrist, and Michael Gar Chung Yiu, Psychiatrist, United Christian Hospital, Hong Kong; email:wmc009@ha.org.hk

1 Common Mental Disorder Clinic, Kowloon West Cluster. Guideline for Common Mental Disorder Clinic Cross-cluster Referral. HAHO-COC-GL-Psy-010-V1.

2 Park M, Unützer J. Geriatric depression in primary care. Psychiatr Clin North Am 2011; 34(2): 469-87.

3 Givens J, Datto C, Ruckdeschel K, Knott K, Zubritsky C, Oslin DW, et al. Older patients' aversion to antidepressants: a qualitative study. J Gen Intern Med 2006; 21: 146-51.

doi:10.1192/bjb.2018.13

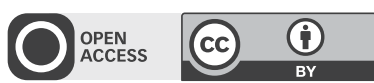

(c) The Authors 2018. This is an Open Access article, distributed under the terms of the Creative Commons Attribution licence (http://creativecommons.org/ licenses/by/4.0/), which permits unrestricted re-use, distribution, and reproduction in any medium, provided the original work is properly cited. 